\title{
EDITORIAL
}

\section{Invited papers from the international meeting on 'New Frontiers in Numerical Relativity' (Albert Einstein Institute, Potsdam, Germany, 17-21 July 2006)}

Traditionally, frontiers represent a treacherous terrain to venture into, where hidden obstacles are present and uncharted territories lie ahead. At the same time, frontiers are also a place where new perspectives can be appreciated and have often been the cradle of new and thriving developments. With this in mind and inspired by this spirit, the Numerical Relativity Group at the Albert Einstein Institute (AEI) organized a 'New Frontiers in Numerical Relativity' meeting on 17-21 July 2006 at the AEI campus in Potsdam, Germany.

It is an interesting historical remark that the suggestion of the meeting was first made in the late summer of 2005 and thus at a time that for many reasons has been a turning point in the recent history of numerical relativity. A few months earlier (April 2005) in fact, F Pretorius had announced the first multi-orbit simulations of binary black holes and computed the waveforms from the inspiral, merger and ring-down ('Numerical Relativity', Banff International Research Station, Banff, Canada, 16-21 April 2005). At that time, the work of Pretorius served as an important boost to the research in this field and although no other group has yet adopted the techniques he employed, his results provided the numerical relativity community with clear evidence that the binary black hole problem could be solved. A few months later (November 2005), equally striking results were presented by the NASA Goddard and Texas/Brownsville groups, who also reported, independently, multi-orbit evolutions of binary black holes using numerical techniques and formulations of the Einstein equations which were markedly distinct from those suggested by Pretorius ('Numerical Relativity 2005', Goddard Space Flight Centre, Greenbelt, MD, USA, 2-4 November 2005).

A few months later other groups were able to repeat the same simulations and obtain equivalent results, testifying that the community as a whole had reached comparable levels of maturity in both the numerical techniques and the mathematical methods needed for successful solution of the Einstein equations for binary black holes. Clearly, an important frontier, and actually a long-awaited one, was finally open and the 'gold rush' was just about to begin by the time the 'New Frontiers in Numerical Relativity' meeting started its sessions in July 2006.

And so, almost 20 years since the almost homonymous meeting held at UrbanaChampaign ('Frontiers in Numerical Relativity', University of Illinois, IL, USA, 1988), the 'New Frontiers in Numerical Relativity' meeting at the AEI saw the enthusiastic participation of a great part of the community, with 127 participants present (in 1988 they were 55) and with a large majority being represented by students and postdocs, a reassuring sign of good health for the community. Faithful to the title of the conference, the programme was dedicated to the many and diversified 'frontiers' in numerical relativity and organized so as to have few talks with ample time dedicated to discussions.

Overall, the talks presented at the meeting covered all of the most salient aspects of numerical relativity: from the formulation of the Einstein equations, over to the initial-value problem in general relativity, from the evolution of vacuum and non-vacuum spacetimes, to multiblock adaptive mesh-refinement techniques, from boundary conditions and perturbative methods, to relativistic fluids and plasmas. The contributions in this special issue represent a 
selection of that research, but also include invited papers from authors who were not present at the meeting but were pursuing research at the forefronts of numerical relativity.

In addition to the more traditional sessions, the 'New Frontiers in Numerical Relativity' meeting also hosted a less traditional session, dedicated to an 'unconstrained' discussion which covered some of the most controversial issues that emerged during the conference. During this session, chaired by E Seidel, a lively discussion took place in the non-trivial attempt of marking the new frontiers on the map of numerical relativity. The transcript of this discussion is an integral part of this issue and it is available, along with the audio recording, in the online version (see stacks.iop.org/CQG/24/i=12). We believe they embody an important part of the development of this field and, like a good bottle of wine, it will be interesting to read them again once sufficiently aged.

As a concluding remark we note that it is almost one year since the "New Frontiers in Numerical Relativity' meeting and dozens of excellent papers have been published or posted on the preprint archive. Some of the scientific results obtained over these months, especially those revolving around binary black holes, were simply unimaginable a few years ago and represent an indisputable evidence that the research in numerical relativity has never been as exciting as it is now.

These results have already had an impact in astrophysics and the community interested in the analysis of gravitational-wave data, thus opening new and different frontiers in numerical relativity. Interestingly, all of this is happening while ground-based gravitational wave detectors in the US and Europe are operating at a sensitivity such that gravitational radiation may soon be directly detected.

While much still needs to be understood and improved, the gold rush towards the new frontiers of numerical relativity does not yet show any sign of being close to a rapid end.

M Campanelli, Center for Computational Relativity and Gravitation, Rochester Institute of Technology, New York, USA

L Rezzolla, Albert Einstein Institute, Max-Planck-Institute for Gravitational Physics, Golm, Germany

\section{Guest Editors}

